\title{
Pour une culture pratique de la confiance, utopie concrète
}

\section{Introduction}

Introduction. Towards a practical culture of trust, a concrete utopia

Introducción. Para una cultura práctica de la confianza, utopía concreta

\section{Laurence Cornu}

\section{OpenEdition}

Journals

Édition électronique

URL : https://journals.openedition.org/ries/5495

DOI : $10.4000 /$ ries.5495

ISSN : 2261-4265

\section{Éditeur}

France Education international

\section{Édition imprimée}

Date de publication : 1 septembre 2016

Pagination : $37-55$

ISBN : 978-2-85420-611-1

ISSN : $1254-4590$

\section{Référence électronique}

Laurence Cornu, "Pour une culture pratique de la confiance, utopie concrète », Revue internationale d'éducation de Sèvres [En ligne], 72 I septembre 2016, mis en ligne le 01 septembre 2016, consulté le 01 juillet 2021. URL : http://journals.openedition.org/ries/5495; DOI : https://doi.org/10.4000/ries. 5495 


\title{
Confiance, éducation et autorité
}

\author{
Introduction \\ Pour une culture pratique de la confiance, utopie concrète
}

Laurence Cornu

Université de Tours EES

Pas d'éducation sans autorité, ni sans confiance. Ce sont là des lieux communs, et l'on pourrait s'attendre ici à lire un dossier au propos évident. Or ce que l'on découvre est inattendu, d'une grande diversité en même temps que d'une commune acuité, et offre une véritable mise en travail de la notion faussement limpide de confiance, centre de gravité du dossier - objet de gravité.

S'il n'y a pas d'autorité sans confiance, la réciproque n'est pas nécessairement vraie. Qu'en est-il dans le champ de l'éducation ? Quelle place y prend la confiance? On attend des enfants qu'ils aient confiance dans les personnes dépositaires de l'autorité à leur égard, en vue de leur éducation. Mais comment se présentent autorité et confiance de par le monde, dans les (dés)orientations contemporaines de l'éducation? Telle était la proposition de réflexion adressée aux auteurs sollicités. La note de quête suggérait trois observatoires possibles de la place de la confiance, de ses formes ou son manque : la classe, les établissements, les systèmes éducatifs.

\section{Diversité de SITUATIONS ET D'APPROCHES, COMMUNAUTÉ D'ATTENTION}

On découvrira donc d'abord dans ce dossier une diversité recherchée de situations selon les pays : Angleterre, Bénin, Brésil, Cambodge, Chili, Djibouti, Finlande, France, Nouvelle-Zélande, Portugal. Tous les articles ont en commun de proposer une mise en perspective historique de la configuration de l'autorité et de la confiance propres à un pays, ce qui dessine diverses figures du « rapport à l'école ". Il en ressort que chaque pays se confronte à la crise de l'autorité, ainsi qu'aux défauts de confiance : on voit à quel point le champ pédagogique n'est pas indépendant du social, du politique et de ses événements, à quel point la confiance y est tributaire de ses épreuves contemporaines. On sait bien que la mondialisation pose ses défis à l'éducation, même si chaque pays la rencontre à son heure et s'y attelle à sa manière. Il est intéressant que cela apparaisse aussi nettement à propos de l'autorité, mais plus encore de la confiance. 
L'autre diversité est celle des approches : les sciences de l'éducation sont présentes, mais aussi l'anthropologie, la sociologie, la psychologie et la philosophie. Or tous les auteurs ont en commun de s'être explicitement confrontés à plusieurs difficultés épistémologiques et méthodologiques de définition de la confiance et de saisie de ses manifestations empiriques : observer - mais quoi et comment ? La confiance se mesure-t-elle ? On constatera une véritable ingéniosité des stratégies de recueil de données : entretiens croisés, recherche d'indices dans les dispositifs, ou d'observatoires indirects à partir de ce qui ruine la confiance ou discrédite l'autorité (les abus, les violences, la corruption, la vénalité de professeurs, la déception...) -, chaque fois précédés de précisions définitionnelles.

Car il ne suffit pas de nommer la confiance pour en saisir le phénomène ; plus même : elle peut être présente dans être nommée (et inversement). Il ne suffit pas non plus de la nommer pour la définir. Comme l'air qu'on respire est la confiance : on la reconnaît vitale quand elle manque. Et on la croit transparente, quand elle relève d'une météorologie complexe. La précieuse bibliographie de Bernadette Plumelle permet de le constater, en même temps qu'on y découvre l'intérêt renouvelé pour cette notion décisive à l'école. La confiance défie les définitions : tous les auteurs s'y sont mesurés. Ainsi, dans la diversité des approches, une autre surprise est que les articles présentent des traits que l'on pourrait dire philosophiques : travail conceptuel, mise en problème, affirmation de thèses engageant leurs auteurs.

\section{L'ÉLABORATION DE DISTINCTIONS CONCEPTUELLES}

\section{Éducation : des enjeux}

Frappante est, à travers les articles, une intéressante moisson de définitions des trois notions. Clarisse Napporn (Bénin) écrit ainsi :

L'éducation ... consiste pour les adultes à créer des conditions permettant aux plus jeunes de développer toutes leurs potentialités. Une bonne part ... se déroule dans les établissements scolaires, où les enfants sont confiés aux maîtres.

Rachel Solomon Tsehaye (Djibouti) en donne une caractéristique :

L'éducation, à travers son outil privilégié qu'est l'institution scolaire, permet à la société d'exercer un contrôle sur les nouvelles générations...

Sans doute une approche sociologique insiste-t-elle sur les déterminismes, et une certaine psychologie - comme la pédagogie - peut-elle insister sur le développement des potentiels. Mais on voit la tension possible et la dimension politique de la discussion : l'orientation de telle éducation est-elle la continuation d'une tradition ou le développement de potentiels inattendus? Visée de contrôle, de productivité, ou d'émancipation? En tout état de cause, 
cette action des adultes auprès des enfants qui leur sont confiés, et qu'on appelle éducation, suppose que les adultes se portent garants de ce qui est à transmettre, et des potentiels des "nouveaux» (Arendt, 1989), et c'est ce qu'on appelle autorité.

\section{Des formes d'autorité}

Le fait que la démocratie conteste l'autorité traditionnelle met au défi l'éducation - seul lieu où elle semble encore appelée, quoique chahutée - d'en trouver de nouvelles formes. Une crise, nous a appris Hannah Arendt, donne l'occasion de revenir " aux choses mêmes » et, en l'occurrence, de mieux définir l'autorité, c'est-à-dire de la distinguer non seulement de l'autoritarisme, mais de la notion de pouvoir, contre un usage commun approximatif qui les assimile. L'autorité est une relation fondée sur une reconnaissance : elle n'existe que si elle est reconnue. Elle est ruinée s'il y a contrainte - et même persuasion (Arendt, 1989). On peut en distinguer des types, travaillés depuis longtemps par la sociologie et la philosophie. ${ }^{1}$

La fin de l'autorité traditionnelle, qui peut ouvrir le champ aux pouvoirs et aux abus de pouvoirs, rend urgent et impératif de préciser ce qui fait le principe de l'autorité en éducation :

L'autorité éducative, il ne faut jamais l'oublier, doit être au service des enfants et s'il y a un pouvoir - des enseignants ou des parents -, ce pouvoir sera toujours, par son statut anthropologique, un pouvoir transitoire, dont la finalité, à la différence du pouvoir politique, se réalisera seulement dans la mesure où son exercice vise et réalise son dépassement: en faveur de la réalisation de l'enfant comme une personne de droits. (Adalberto Dias de Carvalho et Nuno Fadigas, Portugal)

Une telle conception de l'autorité en éducation impliquerait alors une nouvelle importance de la confiance, dans de nouveaux registres : envers les enfants, entre les adultes.

\section{De quelles confiances parle-t-on?}

Quant à la confiance, il faut aussi en préciser le sens et prêter attention à ses formes. La distinction entre confiance relationnelle et confiance politique, que propose José Weinstein (avec Dagmar Raczynskiet Macarena Hernández, Chili), pour dépasser les «lieux communs », a une portée éclairante qui permet d'organiser l'enquête.

\footnotetext{
1. On pense à Weber, à Kojève... Plus récemment : « Il y a une autorité de type autoritaire tout comme une autorité de type démocratique, une autorité à caractère bureaucratique, fondée sur le respect de procédures, tout comme une autorité à caractère professionnel, fondée sur les qualifications et la compétence, une autorité appuyée sur une hiérarchie tout comme une autorité à caractère plus directement relationnel. " Jacques Coenen-Huther (2005): "Pouvoir, autorité, légitimité », "En marge d’un livre récent d’Alain Renaut », (La fin de l'autorité), Revue européenne des sciences sociales XLIII-131. Sur une autorité émancipatrice, voir aussi les travaux de Beatriz Greco (Argentine).
} 
La première renvoie au sentiment éprouvé par les enseignants à l'égard des différents acteurs avec lesquels ils sont en relation quotidiennement à l'intérieur de l'école, tandis que la seconde fait référence à la confiance qu'ils ressentent à l'égard des institutions éducatives et d'autres institutions ou acteurs-clés de la société chilienne.

Sentiment qu'éprouvent les enseignants, dans la classe, ou dans (à l'intérieur) l'institution : Marc Bray, Junyan Liu, Wei Zhang, Magda Nutsa Kobakhidze (Cambodge) en dessinent l'envers. Comme en écho, Denis Meuret (France) distingue confiance relationnelle et confiance institutionnelle, tout en indiquant ceci :

La confiance est un bien public de plus en plus important dans les sociétés modernes, dans lesquelles les interactions avec des personnes inconnues ou peu connues se multiplient.

Ce point est décisif : si la confiance est un bien public, c'est qu'elle est un rapport avec l'inconnu, qui ne refuse pas l'incertain, rapport indispensable dans la modernité. Comme sentiment de non-inquiétude, il y en a différents objets et registres. Le fait de les distinguer dans des concepts donne des outils plus précis d'analyse. Depuis longtemps, la sociologie en a proposé des types, ainsi de la confiance familière, de la confiance assurée (dans un système, une institution - en anglais : "confidence »), et de la confiance décidée ( trust») de Niklas Luhmann (2000), qui définissait la confiance comme un "mécanisme de réduction de la complexité sociale ». La confiance ne s'embarrasse pas de tout contrôler, parfois à tort, mais aussi souvent à raison. Sans elle, la vie courante serait invivable. La confiance familière est vitale, elle est bien un «sentiment de sécurité d'une personne à l'égard de quelqu'un ou de quelque chose ${ }^{2}$. Le "simple bon sens" montre que l'enfant a besoin de cette sécurité sur laquelle il doit pouvoir compter ( to rely on $»$ ). On pourrait dire que les dépositaires de l'autorité sur les enfants ont en charge non seulement la transmission mais cette sécurité, condition indispensable des processus d'apprentissage. Elle est déterminante pour que l'on puisse avoir confiance dans les institutions ( confidence»), ce qui est décisif pour leur bon fonctionnement ${ }^{3}$. Or tous les articles rencontrent le fait que ce qui semble aller de soi n'a rien d'assuré.

À quoi tient la confiance, dont on peut avoir le sentiment encourageant ou l'amère déception ? Mere Skerrett et Jenny Ritchie (Nouvelle-Zélande maorie) en analysent une tradition et sa trahison. Sylvio Gallo et Alexandre Filordi de Carvalho (Brésil) décrivent la dynamique d'une confiance politique réinventée, Irmeli Halinen, Hannele Niemi et Auli Toom (Finlande) la présentent comme une culture, une philosophie même : de la confiance, il y a des événements et des processus, des cercles vertueux, mais aussi des ruines, des inventions, des « régimes»(Cornu, 2005), qu'on peut relier aux formes d'autorité. Rachel

2. Définition du Dictionnaire Larousse, citée par Adalberto Dias de Carvalho et Nuno Fadigas.

3. C'est ainsi que l'Ontario a fait une priorité de l'accroissement de la confiance du public dans les écoles publiques :

cf. http://www.edu.gov.on.ca/fre/parents/IntroDocFre.pdf 
Solomon Tsehaye (Djibouti) distingue ainsi une autorité éducative rationnelle et responsabilisante et une autorité personnifiée, corrélatives d'une confiance relative et d'une confiance absolue.

Toutes ces distinctions (formes, objets, modalités, régimes) sont utiles pour entreprendre d'objectiver ce qui se donne comme "confiance ", mais elles sont aussi appelées par le diagnostic de situations singulières, et par une inquiétude partagée, quoique différemment interprétée par les auteurs. Le problème est alors celui-ci : peut-on analyser et interpréter le fait que le monde contemporain se trouve au défi d'inventer de nouvelles formes de confiance dans le champ de l'éducation?

C'est au regard de ce problème que nous présentons maintenant les articles. La liberté du lecteur est toujours, à loisir, de cueillir à son gré. La succession choisie a été pensée comme un parcours, offrant une mise en problème de la fausse simplicité de «la confiance».

\section{LE PROBLÈME : \\ LE MONDE N'A PAS \\ LA CONFIANCE TRANQUILLE}

La démocratie suppose des citoyens ayant appris à se faire confiance. L'exercice de l'autorité éducative s'en trouve transformé. Mais au delà de la crise de l'autorité, les crises de la confiance, au défi d'être horizontale et non plus verticale, font réfléchir à l'éducation comme bien public.

\section{Démocratie, autorité et confiance}

L'article concernant le Portugal, qui ouvre ce dossier, montre d'entrée le défi posé à la démocratie : celle-ci, récusant les modèles politiques autoritaires, suppose une confiance entre les citoyens, tout en étant aussi confrontée à la concurrence des pouvoirs. La difficulté de l'éducation démocratique est qu'elle doit concilier le respect des "droits libertés » reconnus aux enfants avec le devoir de les protéger dans leurs «droits créances». Mais selon quel exercice de l'autorité ? Si la finalité d'une éducation démocratique est de rendre vivante une confiance entre égaux, alors l'autorité est temporaire, et son exercice est transformé : c'est aux maîtres de faire confiance à quelque chose des initiatives des enfants. Ainsi la pédagogie, sans aucunement renoncer à son rôle de garant (auctor), s'ouvre sur des formes nouvelles, impliquant la coopération des élèves, la pratique de projets. Philosophe attentif à ce qui s'est inventé en pédagogie, Adalberto Dias de Carvalho montre avec Nuno Fadigas, que dans la visée démocratique, il est décisif que les élèves apprennent l'autonomie et la confiance en en faisant l'expérience. Au Portugal, une série de dispositions réglementaires ou légales a favorisé (sans la nommer) cette confiance qui mène à l'autonomie, mais il y a aussi des retours en arrière. Ce sont alors des pédagogies originales qui soutiennent ce pari. 
Car rien n'est facile dans ce pari, et même si un système démocratique est choisi, comme ce fut le cas au Bénin en 1990, il faut des années pour mettre en place les conditions d'une confiance dans la relation maître-élève, rongée par des abus. En arrière-fond, il y a la rupture avec un modèle traditionnel et une décennie d'incurie dans les recrutements, dans une période de "régime révolutionnaire» (1972-89). On y voit clairement le conflit entre des formes anciennes d'éducation, visant à perpétuer une discipline morale servant une hiérarchie traditionnelle, et des aspirations démocratiques, fragilisées par le manque de formation des enseignants, d'où le développement de "fléaux tels que la corruption, le harcèlement et les abus d'autorité », qui «ont toujours existé, mais ont connu un accroissement au cours de cette période ". D'où la ruine de toute confiance dans l'école. Outre un travail définitionnel attentif sur la notion d'autorité, l'article fait état d'une importante et récente enquête par entretiens. La recherche est par elle-même une forme d'action: Clarisse Napporn y cherche des points d'appuis pour lutter contre abus et incuries, et construire avec les acteurs eux-mêmes une confiance à l'intérieur des classes et dans l'institution.

\section{COMMENT MESURER LA CONFIANCE ET À QUELLES CONDITIONS LA SOUTENIR À L’ÉCOLE?}

Que l'on ne croie pas qu'il s'agisse là du problème de " jeunes » démocraties. Celle-ci est toujours à réinventer, comme l'article sur la France proposé par Denis Meuret le donne à penser. L'on voit ici problématiser la difficulté de cerner et d'objectiver quelque chose de tel que "la confiance". Explorant plusieurs études sur la défiance, comme la diversité des définitions de la confiance, l'auteur rappelle le diagnostic selon lequel il y a en France une défiance importante, et une défiance envers l'école dont l'ampleur n'est pas justifiée par le taux de corruption corrélé dans d'autres pays. Quelle que soit l'interprétation, il y a deux aspects du problème : «l'expérience scolaire détermine à la fois la confiance au sein de l'école et, partiellement, la confiance sociale. " L'auteur, là encore, s'engage, en soutenant une "expérience scolaire qui favorise la confiance au sein de l'école ", car elle est nécessaire aux apprentissages, et une expérience scolaire qui, d'autre part, "éduque à faire confiance, dans une société où il peut arriver qu'elle soit déçue. » La confiance est question d'expérience; il faut « l'accorder à bon escient». Mais "comment faire confiance à une école qui prêcherait la confiance, mais ne ferait pas elle-même confiance à ses acteurs (professeurs et élèves) ?»

La confiance est décidément bien difficile à établir - et d'abord à saisir.

José Weinstein et al., analysant la situation d'écoles de Valparaiso, proposent une étude pionnière, qui se confronte à la difficulté de "mesurer » la confiance, notion trop vague. D'où le choix de deux types de confiance, la 
confiance relationnelle et une confiance politique. Où l'on apprend que ce qui se passe dans la classe (la confiance, confirmée comme condition des apprentissages, avec cinq dimensions fondatrices ${ }^{4}$ ) est bien inséré dans un réseau de confiances entre adultes (collègues, direction, parents), confiance institutionnelle elle-même située dans un dispositif politique. La confiance en interne du cadre éducatif a donc un contexte extérieur. Il se révèle des confiances multiples, dont certaines non corrélées. On peut imaginer, d'ailleurs, que la toile de fond est aussi, au Chili, son histoire politique, et sa sortie de la dictature, vers la démocratie. Si « les confiances des enseignants chiliens révèlent des obstacles importants - comme le manque de confiance à l'égard des parents, des élèves ou des autorités éducatives ", là encore les auteurs sont engagés, acteurs par leur étude même (qui aura des prolongements).

\section{Histoire longue de tensions contemporaines et recherche de nouvelles voies}

Portée de l'histoire et question de la confiance faite aux parents : l'article sur Djibouti de Rachel Solomon Tsehaye met en évidence les tensions de l'après colonialisme et celles d'un fondamentalisme islamiste. La confiance relationnelle et la confiance institutionnelle apparaissent elles-mêmes enchâssées dans ce que l'auteur appelle les "systèmes mondes », ensemble d'actes de foi par lesquels on assoit une confiance dans le monde : à Djibouti, se heurtent un système-monde occidental et un système-monde oriental. Cette étude très ingénieuse montre que l'indication significative est non seulement ceux à qui, mais ce dans quoi on fait confiance. La confiance dans l'école, à l'occidentale, suppose la confiance dans les savoirs.

[Or] l'islam dogmatique est contrarié par l'individualisme moderne, le matérialisme inhérent à l'industrialisation et le paradigme scientifique qui suppose d'avancer dans l'incertitude, de douter et d'avoir confiance en la raison critique.

Mais l'auteure montre aussi que le modèle occidental, loin d'être « innocent ", est susceptible de reconduire le colonialisme. Construisant un tableau très éclairant des "rapports à l'école ", elle affronte à son tour le défi contemporain d'une "perte de confiance liée à l'incompréhension de l'usage qui peut être fait de savoirs scolaires parcellisés et décontextualisés ", et l'ouverture d'une troisième voie. Elle engage même à soutenir une « utopie» :

Changer le rapport à l'école et donner la possibilité aux élèves de participer concrètement et activement à la construction de l'espace qui les forme, apparaît être une initiative utopique (mais non illusoire) et prometteuse : la prise en compte des subjectivités, des géographies occupées et des histoires biographiques des individus aurait pour effet vertueux de [...] pallier la crise du sens.

4. Dimensions qui permettent la relation, d'interdépendance et de «trust » : bienveillance, honnêteté, ouverture, fiabilité, compétence. 
Que la confiance dans l'éducation soit investie de la mémoire des trahisons de confiance dont l'impérialisme occidental a été capable, c'est ce qui est sensible dans le texte consacré à l'éducation maorie en Nouvelle-Zélande. La lutte pour la reconnaissance passe par la reconnaissance de l'éducation ancestrale, très en lien avec le rapport au milieu naturel, et faisant place à l'expérience, une expérience "parlée » dans un langage poétique, une mythologie vivante. Le lecteur étranger, au delà d'une «identité » non partageable, y entend un message qui lui parle : d'une part, il est question d'une éducation qui respecte les enfants. On peut voir dans l'illustration présentée (un enfant encore relié par son cordon et porté par un canoë à tête d'animal) comme une métaphore du holding de Winnicott. Et éduquer, c'est alors - métaphore suggestive ! - « choisir la vague sur laquelle lancer le canoë... ». D'autre part, l'importance de relier les acquisitions de savoir à une expérience qui fasse sens dans des " contextes authentiques " est une suggestion précieuse.

La rationalité des savoirs est leur force (et leur fiabilité vient d'une méfiance rationnelle), mais leur transmission souffre de leur émiettement ou de la négligence de ce qui pourrait faire sens, et qu'il faut disputer aux dogmatismes et aux crédulités. Pas d'éducation sans la question d'un sens, saisi dans des fiabilités concrètes, humaines et pragmatiques, confiance possible dans le monde, dans un sens du monde qui suppose des formes de confiance et de lien - qui ne sont pas nécessairement religieuses ${ }^{5}$.

\section{La confiance dans l'éducation à l'épreuve du néolibéralisme}

En fait, l'opposition n'est peut-être pas tant entre des "civilisations" ou des cultures, qu'à l'intérieur même de l'Occident, dont l'éducation est insidieusement livrée au néolibéralisme. Pointant les croyances propres au système néolibéral et leurs effets de cercles vicieux, Peter Kelly fait le diagnostic de l'éducation gouvernée par l'évaluation en Angleterre. Il montre comment la mise en place des évaluations ${ }^{6}$ oriente les stratégies des établissements, des enseignants, formate les curricula et jusqu'aux esprits, qui intériorisent ces normes. À l'approche par compétences s'est substituée celle par performance. On "croit » à autre chose : aux chiffres et aux mesures, qui occultent les appréciations, les «jugements directs » que nous pourrions faire sur ce qui vaut la peine d'être transmis et cultivé. Les chiffres sont une croyance, nous leur faisons confiance, mais l'évaluation chiffrée concurrentielle finit par gouverner l'éducation, faisant

5. Même si l'on peut rappeler l'étymologie, fides - foi - de confiance, que l'on retrouve dans fidélité, confidence, fiabilité, fiducie (etc.), on pourrait faire une lecture de la modernité en termes de sécularisation de la confiance dans le monde.

6. Maroussia Raveaud avait déjà noté un changement dans les tendances anglaises, dans les années 2000 : la pédagogie child centered, jusque-là prévalente, est mise à mal par le dispositif des évaluations. Revue internationale d'éducation de Sèvres $n^{\circ}$ 57, dossier « Le plaisir et ennui à l'école », 2007, p. 89. 
négliger des disciplines (lettres, arts...), atteignant la confiance comme sécurité nécessaire aux apprentissages. L'idée selon laquelle une "main invisible » régule l'ensemble est une confiance illusoire : ce qu'elle engendre, ce sont des injustices et un sentiment d'iniquité, qui ruine la confiance dans l'institution, et peut-être même l'éducation.

Où l'analyse des conditions de la confiance dans la relation pédagogique et dans l'institution scolaire devient critique et politique. L'article de Mark Bray et al. sur le Cambodge montre ce qui se passe lorsque le marché prend la main. Se relevant avec difficulté de la tragédie Khmer rouge, le pays reconstruit une éducation, sans pour autant avoir les moyens de payer ses enseignants. Il s'est donc installé au Cambodge une "éducation de l'ombre » dans laquelle les enseignants titulaires dispensent des cours supplémentaires à titre privé et moyennant finances. Le prétexte d'être «plus proches » d'élèves dans des cours en effet moins chargés, parce qu'ils sont réservés aux plus riches, masque mal les jeux de pouvoir et la ruine de la confiance pédagogique, et institutionnelle, opérée par cette perversion. Il apparaît avec clarté que le noyau de la confiance que des élèves peuvent faire à des enseignants (et des parents à l'institution) tient au fait (ici démontré par son absence) que les enseignants soient avec équité au service de l'enseignement de chacun, quels que soient son origine et ses moyens. Cette perversion, nous avertissent les auteurs, peut survenir dans d'autres pays. Est-on donc impuissant devant cette "main invisible» du marché qui exacerbe les inégalités?

\section{Philosophie et politique de la confiance}

L'article sur le Brésil décrit alors la résistance qu'un ensemble d'écoles ont opposée à une réforme que voulait imposer l'État de São Paulo, sans aucune concertation. Les auteurs font usage d'outils intellectuels philosophiques : lorsque les citoyens "assujettis » se sentent trahis dans ce qu'ils peuvent légitimement attendre de l'État en matière d'éducation (une forme de confiance), ils s'inventent citoyens-acteurs. D'assujettis, des êtres, ensemble, se constituent en "groupessujets " : la distinction conceptuelle entre groupe sujet et groupe assujetti, ici reprise de Deleuze et Guattari par Silvio Gallo et Alexandre Filordi, montre une ressource de la philosophie quand il s'agit de penser les métiers "impossibles ", c'est-à-dire ceux où l'on agit avec autrui. Quand une confiance vient à manquer dans une "gouvernementalité » (autre concept cette fois emprunté à Michel Foucault), il arrive que des êtres aient le courage de la réinventer. En se montrant acteurs, les êtres révèlent les sujets qu'ils sont, en même temps qu'ils font confiance à d'autres «sujets": voilà le "groupe sujet » qui s'éduque lui-même (ou se soigne). «Groupe sujet » (plutôt qu'assujetti), cette création conceptuelle, philosophique, permet d'accueillir le fait que dans la déception d'une confiance (l'échec d'une gouvernementalité), le souci partagé de la préservation de la confiance de base que requiert l'éducation peut donner lieu à l'invention d'une 
confiance politique "autogérée ». Remarquons, dans le pays de Paolo Freire, de la pédagogie de la libération, qu'une tradition anarchiste, en constituant l'éducation en bien public, retrouve l'archè de la chose publique : l'enjeu de l'éducation mobilise des citoyens à la réinventer.

Remarquons aussi que les notions philosophiques de ce texte peuvent permettre une relecture des textes précédents : c'est une gouvernementalité que met en lumière Peter Kelly. Dans la mention répétée de l'acceptation passive de directeurs d'écoles, au Cambodge, on peut voir aussi le fonctionnement de "groupes assujettis ». Les « coopératives parentales", initiées par des femmes maories, s'opposant à "l'assujettissement des enfants» sous des normes standardisées, manifestent des groupes-sujets. On pourrait aussi lire les descriptions du système finlandais d'éducation comme l'action de "groupes sujets ", dans les classes, les établissements, les municipalités mêmes.

L'article finlandais insiste sur le fait que la confiance est bien un travail collectif, que la conscience de l'importance du climat de classe, de la participation des élèves implique tous les acteurs. On y voit des caractéristiques de la confiance pédagogique, mais aussi que la recherche commune aux adultes garants des conditions optimales d'apprentissage, mobilise entre eux des « coopérations multiples », de relations avec les municipalités, de dialogue dans les réformes, et de «confiance mutuelle ». On est tenté d'y voir l'utopie concrète appelée par Rachel Solomon Tsehaye, et sa dynamique vertueuse d'auto-renforcement. Il ne faut pas négliger qu'elle puisse être aussi menacée par les coupes budgétaires: elle requiert un travail collectif au long cours. La confiance se construit, dans l'expérience, la coopération, dans des opportunités saisies, avec du temps. On voit ici la place des parents, en qui l'on a aussi confiance, et l'importance décisive de la formation des maîtres, thématique présente dans d'autres contributions. Il s'agit d'une véritable "culture de la confiance », et même, disent les auteurs, d'une "philosophie» de la confiance : la confiance y devient bien un mode opératoire - comme annoncé par Adalberto Dias de Carvalho.

Des concepts philosophiques "concrets ", " opératoires » : c'est de cela qu'il s'agit dans la confiance. Y aurait-il dont un lien entre philosophie et confiance?

\section{LA CONFIANCE COMME PROBLÈME PHILOSOPHIQUE}

\section{Des thèses convergentes}

Il se dégage plusieurs thèses convergentes sur la confiance comme une condition « sans laquelle il est difficile (voire impossible) d'atteindre une gestion éducative de qualité entre les différents acteurs qui la constituent. »(Chili).

L'école fonctionne mieux si ses acteurs (élèves, enseignants, parents, chefs d'établissement, etc.) se font confiance. (Denis Meuret). 
Si l'école a besoin de confiance relationnelle, pour les apprentissages, il s'agit de s'ouvrir à des pédagogies plus attentives et participatives. À son tour, la confiance relationnelle a besoin de confiance institutionnelle, pour que les responsables eux-mêmes puissent assurer leur travail. L'espace de confiance de la classe, espace de la relation maître élève et de son climat de sécurité, suppose la confiance - cette fois au sens de l'espérance - qu'une société met dans l'école et dans ses responsables, et implique encore la confiance commune placée dans des valeurs et des savoirs. Mais les auteurs sont inquiets de ce que le simple bon sens ne fasse pas sens commun. Aussi vont-ils plus loin en posant des jugements directs, et en formulant des propositions originales, qui comportent toutes la reconnaissance des efforts que chaque pays a à faire, marquant ainsi le sens de leur engagement de chercheurs, allant bien au delà des objectivations construites, affirmant qu' « un autre avenir est possible» (Peter Kelly).

\section{L'engagement des auteurs}

La lecture de l'ensemble de ces articles nous indique qu'il ne s'agit pas seulement de la confrontation de la démocratie politique avec l'autorité et la tradition, mais du danger que le marché, c'est-à-dire ici la marchandisation de l'éducation, fait courir non pas à tel ou tel pays, mais à chacun d'eux.

L'urgence est de produire des horizons servant à vaincre le sentiment d'arbitraire, d'efforts vains et d'inutilité, en luttant contre l'autoritarisme contreproductif exercé par l'imposition normative d'un modèle éducatif, la parcellisation des savoirs scolaires et les pratiques en classe. (Rachel Solomon Tsehaye).

Nous pourrions, au lieu de transformer en biens marchands des élèves dont on dédaigne la profondeur et la complexité, célébrer au contraire les diverses formes de réussite de tous nos enfants à mesure qu'ils évoluent dans le système scolaire jusqu'à l'âge adulte. Le jeu en vaut assurément la chandelle. (Peter Kelly)

Ainsi, non seulement les auteurs ont défini des concepts, et les ont fait jouer dans des problématisations, mais chacun s'est engagé dans des thèses anthropologiques, sociologiques, politiques, posant des jugements directs. Il me semble que ce dossier propose comme une philosophie concrète de la confiance, nourrie d'études, posant des diagnostics, soutenue par quelques concepts philosophiques opératoires, et engageant à des pédagogies ouvertes aux processus de confiance émancipatrice (Cornu, 2006). La confiance est un mode d'action avec autrui. Comme culture, elle est une certaine disposition d'esprit dans l'action, disposition à la coopération. Elle est une acceptation de l'inconnu de l'autre. Elle ouvre des possibles. C'est une "mise », et le fait de faire cette mise, même minime, a des effets décisifs, parce que cela engage une relation d'une certaine manière, comme un don qui laisse venir le contre-don. La confiance nécessaire à l'enfance repose sur l'engagement de confiances entre les adultes. Qui fait la première mise? "Souhaitons-nous d'être inventifs ${ }^{7}$."

7. Rachel Solomon Tsehaye. 


\section{BibliographiE}

ARENDT H. (1989) : "La crise de l'éducation », in La crise de la culture, Folio.

CORNU L. (2006) : "La confiance comme relation émancipatrice ", Les moments de la confiance, sous la direction d'Albert Ogien et de Louis Quéré, Economica.

CORNU L. (2005) : "Autorité, hospitalité", in Jacquet-Francillon François et Kambouchner Denis (dir.). La Crise de la culture scolaire: origines, interprétations, perspectives, Paris : PUF.

CORNU L. (1998) : "La confiance dans la relation pédagogique », Le Télémaque, $\mathrm{n}^{\circ} 13$, mai, PUC.

DONZELOT J. (2004) : Faire société. La politique de la ville aux États-Unis et en France, Seuil.

LUHMANN N. (2006): La confiance, un mécanisme de réduction de la complexité sociale, Paris Economica.

OGIEN A, QUÉRÉ L. (2006) : Les moments de la confiance, Economica.

SHEETS-JOHNSTONE M. (2006) : «Sur la nature de la confiance », dans Les moments de la confiance, op. cit.

SIMMEL G. (1991) : Secret et sociétés secrètes, Circé.

WATIER P. (2008) : Éloge de la confiance, Belin.

WINNICOTT D.W. (1993) : L'enfant et sa famille, Payot. 